\title{
Coordinative Inference Rate Maximization In MIMO-Visible Light Communication
}

\author{
Dasari Subba Rao ${ }^{1}$, N.S. Murti Sarma ${ }^{2}$ \\ ${ }^{1}$ Research Scholar, Rayalaseema University, Kurnool, A.P., India \\ ${ }^{2}$ Professor of ECE, Sreenidhi Institute of Science \& Technology, Hyderabad, India.
}

\begin{abstract}
In the propagation of signal over channel using optical modulation, the observed interference constraint the transmission efficiency. In a visible light communication model, Multi input multi output (MIMO) based communication models were used for its higher throughput and spectrum utilization. In MIMO based visible communication model, the objective of maximizing rate allocation for optimal transmission is focused. In the conventional model, the rate allocation were performed based on the traffic model, and no interference consideration were made. This constraint the optimization of resource allocation in MIMO based visible light communication. in this paper, a new approach for resource allocation based on dual metric consideration of traffic consideration and inference model is developed.
\end{abstract}

Keywords: MIMO system, visible light communication, propagation model, rate maximization problem.

\section{Introduction}

Wireless channel are getting diverse in nature. The uncertainty in channel conditions has degraded the estimation performance of conventional channel estimator logic and needs an updation. The feedback estimators are hence developed to achieve the estimation performance under variant channel condition. Among various approaches of channel estimation logic, feedback based estimators are used as a feedback estimator, which works on the objective of channel tracking. The estimation process is governed by a state transition logic, where the process of estimation and updation is carried out to obtain a estimate. In the process of MIMO based channel estimation, the application of feedback filters are made for its simpler coding and estimation performance. However, with the increase in communication approaches and offered services, the conventional models of feedback filtration is need to be improved. To derive better performance for such system, different methods were developed in recent past. In [1] a frequency selective fading channel estimation is proposed. the issue of channel imperfection is analyzed. A novel method of pilot expansion is proposed to capture multipath signal arise in the MIMO system. A Decision feedback filter is proposed for channel tracking in communication system. The approach of flexible pilot flexible coding is been presented. A joint estimation of channel gain and phase noise in MIMO system is analyzed in [2]. A decision directed extended feedback filter for phase noise tracking is developed. The approach of extended feedback filter is observed to be more stable in tracking phase noise estimation. A similar approach of channel estimation in semi-blind approach is proposed in [3]. A recursive estimation updation in feedback based estimation logic using blocks for MIMO system is employed. A dynamic block processing expansion model is presented to channel estimation for fast fading channel. The issue of diversity in MIMO system is been analyzed in recent time. In [4] to achieve a faster divergence solution, a channel estimation algorithm based on feedback filter is developed. A state transfer coefficient (STC) is derived with a threshold correction logic for time varying environment. Towards the issue of user mobility, in $[5,6]$ new scheme of mobility concern in MIMO system is suggested. A intelligence logic using fuzzification was developed for variant format of slow, fast and medium speed mobility. The feedback filter is designed to perform the channel estimation for the signal received under mobility condition. The operation efficiency of a channel estimator is dependent on the channel impulse response estimation in MIMO system. In [7] a channel impulse response estimation based on the correlation of transmits or receive antennas. An approach of concatenated wiener filters for the optimization of channel estimation by optimizing the channel characteristic in time and frequency domain. In [8], to estimates channels in different high speed mobiles environments, a wiener filter based approach with basis expansion model (BEM) is presented. The suggestive approach provides a better estimation under time variant channel condition. Towards effective estimation, In [9] a novel a symmetric extension method was suggested for VLC system to reduce the MSE, leakage power and noise of conventional DFT. The estimation of partial frequency response is symmetrically extended as well as reduced MSE and noise eliminated with very small power loss. In [10] a SCM based blind channel estimation method for zero padding MIMO systems have the distinctive features. The identifiability condition is very simple and is more relaxed than the irreducible or column reduced condition. It can apply to the more transmit antennae case under a certain condition. Through numerical simulation, it yields improved BER performance in the low-to-moderate SNR region. In [11] a first order approximation method as well as a second order approximation method for joint 
CFO and CIR estimated in VLC systems. In first order approximation method provides adaptive iteration algorithm with excellent estimation and tracking range compare to the conventional. The Second order approximation method will improves frequency tracking range and channel estimation compare to first order method. In [12] a channel estimator computes the long-term features through a subspace tracking algorithm by identifying the invariant ( space-time modes of the channel. On the other side, the fast-varying fading amplitudes are possibly tracked by using LS techniques that exploit temporal correlation of the fading process. In particular, MIMO system with BICM and MIMO-turbo equalization has been selected as a benchmark for performance evaluation in terms of BER. In [13] a semi-blind timing synchronization and channel estimation scheme for systems was developed based on unit vectors. Semi blind approach having three stages, (i) coarse timing offset with maximum gain is obtained in multipath fading channel, (ii) a fine time adjustment algorithm to find actual time position in channels, (iii) based on final timing estimation obtained frequency-domain in channel response. In this paper we propose a new methodology to assess the performance of MIMO-VLC systems over space frequencies elective channels and non-stationary interference. The performance is evaluated in terms of average bit error probability at the output of the forward error correction (FEC) decoder. We concentrate on convolution coding since this is the mandatory FEC scheme for the majority of the commercial standards based on optical modulation with BICM technology. Nevertheless, the analysis based on the union bound approach is general and can be extended to either convolution or block codes. To provide a brief insight in the proposed methodology, let us consider the transmission of a codeword over a set of parallel sub-channels.

\section{Communication model}

\section{Visible Light Communication}

In MIMO based visible light communication systems, the side information can be the correlation due to the time evolution of the channel, the correlation between channel taps and frequency subcarriers. Many studies exploited time and frequency domain correlation to get the advantages of both domains. With MIMO, correlation from spatial domain exists. Spatial correlation arises due to close antenna spacing's and poor scattering environments. Coherent demodulation of the transmitted symbols requires accurate channel estimation. MIMO channel estimation can performed in frequency and/or time domains. In frequency domain, channels at each subcarrier are estimated. In time domain estimation, the unknowns are the channel length, tap delays, and their corresponding coefficients. Channel estimation methods can be improved by using the side information. In every cell the base station(BS) is equipped with $N_{R} \geq 1$ antennas and each of thesubscriber stations (SSs) has an antenna array of $N_{T} \geq 1$ elements. The transmission is organized according to the logical frame, with $K_{T}$ adjacent subcarriers observed over $Z$ consecutive symbols. Within each cell, multiple accesses is handled by dividing the logical frame into frequency-time units (data regions) of $K \times Z$ subcarriers each. The BS can assign one or more data regions to each SS. The multi-user scheduling strategy provides the mapping rule from the logical frame onto the physical resource to form the time-frequency physical frame. Since some of the subcarriers might remain unassigned, the traffic load $\eta \leq 1$ is introduced to denote the number of active subcarriers out of the total number. Depending on the degree of cooperation among BSs and on the traffic load $\eta$, every data region can experience up to $\mathrm{N}_{\mathrm{I}}$ interferers with constant power over the whole data region. Differently from coordinated approach, the interference randomization policy employs a cell-specific permutation of the subcarriers over the bandwidth before mapping the logical subcarriers onto the physical resources, for the purpose of randomizing the interference within each data region. However, since the optimization of the scheduler is beyond the scope of this paper, the assignments of the data regions are here assumed to be random and independent from cell to cell as for a non-optimized scheduler.

\section{Channel Model}

In frequency-selective multipath environment, the $\mathrm{NR} \times N \mathrm{~T}$ channel response $H_{k}$ on the $i_{\text {th }}$ subcarrier can be modeled as the sum of $\mathrm{W}$ path contributions:

$$
H_{k}=G_{k} \sum_{r=1}^{W} \sqrt{P_{r}} A_{r} \exp \left(-j 2 \pi \frac{k}{N}\right)
$$

Where each path is characterized by mean power $P_{r}$, the NR $\times N$ T fading amplitudes. The complex term $\mathrm{G}_{\mathrm{k}}$ denotes the frequency response of the cascade connection of the transmitter and receiver filters on the ith subcarrier, The fading amplitudes(are assumed to be Rayleigh distributed and uncorrelated from path to path, according to the wide sense stationary uncorrelated scattering model:

$$
R_{S, r}=R_{T x, r} \otimes R_{R X, r}
$$

accounts for the spatial correlation of the fading channel, denoting the Kronecker product and with $R_{T x}$, and $R_{R x}$, being the spatial covariance's among the transmitting and receiving antennas respectively. Following the same reasoning's, we consider two different models corresponding to different assumptions about correlation (2) and geometry of the antenna arrays: a beam forming model is adopted for antenna arrays with closely spaced apart elements and a diversity model for array elements that are sufficiently far apart. In this section we define two specific scenarios for performance assessment. If interference is spatially correlated, the covariance matrix (1) is 
structured and thus the multi-antenna combiner can exploit this knowledge to mitigate the inter-cell interference by an appropriate beam forming strategy.

\section{Interference Coordinated Rate Maximization}

In this article, we have the data rate, delay buffer queues, link quality, and routers, such as the overhead Mac uses standards based hop-hop protocol mode based guidance is suggested. Jam channel high performance with preference given to improving access to links. The proposed routing protocols, the last dimension, a combined weight that is calculated for each node in the assessment of worth. Same source and multiple routing protocols, the basis for choosing the destination of many lines show uncorrelated request. Shown paths between the supply network, each packet from the source node to the destination node of the contract based on the weight, select the path with the lowest cost index. The contract is calculated so that the deployment back. Neighbors of your neighbors downstream nodes potential of cost index feedback response. In order to ensure that the node is increasing and there is a link to the electronic signals that communication on the verge of breaking trust. This signals to measure the impression that it is on the verge of breaking the link is possible, depending on the quality. Therefore, the physical layer using the received signal strength, link quality can be predicted, and select the link path signal strength less than will be rejected. When a transmitting node transmitting the RTS packet transfer, it transmits power Pt. RTS packet is received, the designated node defined free space propagation model measures the strength of the received signal to maintain a relationship with,

$$
P_{r}=P_{t}\left(\frac{\lambda}{4 \pi d}\right)^{2} \cdot G_{t} \cdot G_{r}
$$

where $\lambda$ is the wavelength of the carrier, D is the distance between the transmitter and receiver; GT And has the advantage of GR and antennas transmit, receive, respectively. Is not considered the impact of noise and fading. Therefore, the quality of links, promoting energy [10] is taken as,

$$
L_{q}=P_{r}
$$

To increase performance in heterogeneous wireless networks, a power control mechanism, [11] was proposed. Heterogeneous wireless networks to transmit power by controlling the interference factor was controlled to achieve highest performance. To achieve high performance, low total power allocation for a linear programming model is developed.

Interventions based power management method in [12] at least a minimum total power allocation is defined by power allocation algorithm. A symmetric link to the landscape, link state $C_{i}$, the ' 1 ' is set to be available to link any links to the condition ' 0 ' is. In the case of asymmetrical links, receipt case $C_{j} \neq_{i}$ have not considered. Therefore, the task force disturbances, the transfer of data for $\mathrm{i}-\mathrm{j}>\mathrm{j}$ will not be the same as $\mathrm{P}_{\mathrm{j}, \mathrm{i}}$. Because power is subject to the maximization of the source data rate $\mathrm{R}_{\mathrm{i}}$.

Limited to,

$$
\max \left(\sum_{i \in \text { sources }} p_{r}\right)
$$

$$
\sum_{j \in N_{i}} R_{i j}-\sum_{j \in N_{i+}} R_{j i}=R_{i}
$$

Ni nodes where the 'I' can reach the source and $\mathrm{N}_{\mathrm{i}+}$ nodes that ' $\mathrm{i}$ ' ' is the number of nodes that can reach numbers. In the case of a symmetric link condition, the nodes communicate with a receipt. In this case, there are two types of network nodes, and in that case, $\mathrm{C}_{\mathrm{ij}}=\mathrm{C}_{\mathrm{ji}}$. In such a case, is defined as the level of intervention,

Subjected to,

$$
\max \left(\sum_{i \in \text { sources }} R_{i}\right)
$$

$$
\sum_{j \in N_{i}}\left(R_{i j}-R_{j i}\right)=R_{i}, \forall i
$$

Selecting a link topology that is designed to meet the necessary criteria. The topology of the data on the selected criteria of a transport ban allocated power level will lead to high performance.

For a Quadrature modulation scheme, with $\mathrm{M}$ constellation, the modulation set is defined for the transmitted symbols as,

$$
s_{k}=\left(s_{k}^{Q}+j s_{k}^{I}\right) \sqrt{E_{g}}
$$


where $, s_{k}^{Q}, s_{k}^{I} \in\{ \pm 1, \pm 3, \ldots \pm \sqrt{M}-1\}$, and, $E_{g}=\frac{3}{2(M-1)}$ is the transmitted energy defined for the Mconstellation.

In the process of block coding the bit inter leaver logic is used to control the bit interleave map bits to a constellation symbol on the sub-carrier and to a position in the modulation label set based on the derived bit length.

The inter leaver operation is defined by a set of interleaved data $\mathrm{L}$ represented as, $L=\left\{l_{1}, \ldots ., l_{p}\right\}$ for a given input data, $x=\left\{x_{1}, \ldots, x_{p}\right\}$.

Here, the transmitted symbol $x_{k}$ depends not only on the bit in $l_{k}$ but also on the remaining $m-1$ bits of the label. The symbols are selected by the inter leaver from different positions of the same coded block, leaving the other $\mathrm{m}-1$ bits as independent variables.

Here each bit of the derived error is assigned to a different frequency: $f_{i} \neq f_{j}$ for $i \neq j$ where $\{\mathrm{i}, \mathrm{j}\}=1, \ldots, p$ for any coded bit sequence to be modulated for any given label set $\mathrm{L}$, there are $2^{(\mathrm{m}-1) \mathrm{p}}$ possible symbol sequences $\chi$ with equal probability given by,

$$
P(\chi)=1 / 2^{(m-1) p}
$$

Here, each SINR value $\gamma_{k}$ is scaled by a factor to account forthe Euclidean distance between the transmitted symbol andits nearest concurrent, in the considered symbol constellation $\Delta_{\mathrm{k}}^{2}$. This factor varies with $x_{k}$ and $l_{k}$ and the number of interleave bits, selected to optimize the maximization criteria given by,

$$
\mathrm{L}=\max \left(\Delta_{\mathrm{k}}^{2}\right)
$$

This adaptive controlling, controls the bit allocation in the channel for multiple transmission to the monitoring of symbol correlation, and as the required bit is allocated for the modulation, the error controlling in the communication process is dynamically achieved.

\section{Simulation Results}

The proposed approach is simulated for a MIMO system using visible light communication is developed. Here the transmitting bits are interleaved as per the block length derived from the bit sparse logic. The bit sparsing over the transmitting bit is derived from the interference mode defined. To evaluate the performance of the bit coding, the proposed approach is simulated for different given block length $\mathrm{L}$ and the performance is evaluated for a multipath fading channel with fading factor $\mathrm{K}=30 \mathrm{~dB}$, with Doppler shift $=100 \mathrm{~Hz}$ and AWGN noise of 10dB. The observations obtained from the stated system is as outlined below.

Case I: $K=30 \mathrm{~dB}$, with Doppler shift $=100 \mathrm{~Hz}$ and AWGN noise of $10 \mathrm{~dB}$.

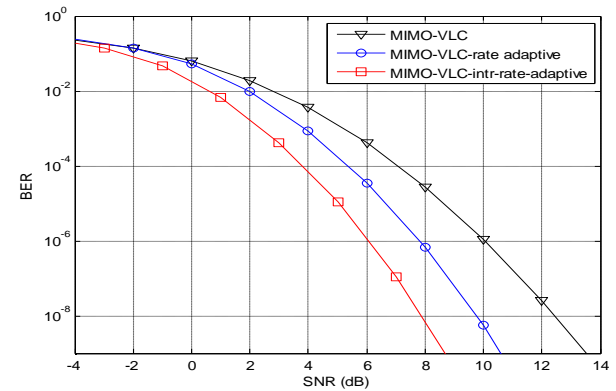

Figure 1 Simulation result of the proposed approach for $\mathrm{L}=3$

The BER performance of the developed system using sparsity coding is evaluated with the conventional approach of block coding. The obtained BER performance with variation in SNR is shown in Figure 1 . The error performance is observed to be minimized by the application of sparse coding over space time coding.

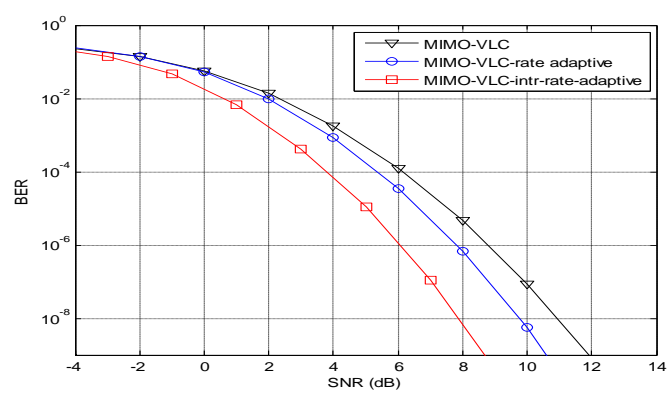

Figure 2 Simulation result of the proposed approach for $\mathrm{L}=6$ 
Case II: $\mathrm{K}=10 \mathrm{~dB}$, with Doppler shift $=100 \mathrm{~Hz}$ and AWGN noise of $10 \mathrm{~dB}$.

A similar case analysis is carried out in different propagation channel conditions, with fading factor $\mathrm{K}=10 \mathrm{~dB}$, Doppler shift of $100 \mathrm{~Hz}$ and Additive White Gaussian Noise (AWGN) of 10dB. The BER observations are shown in Figure 2

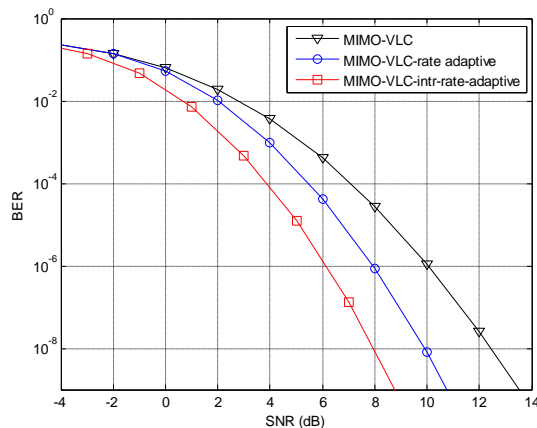

Figure 3 Simulation result for the proposed approach for $\mathrm{L}=3$

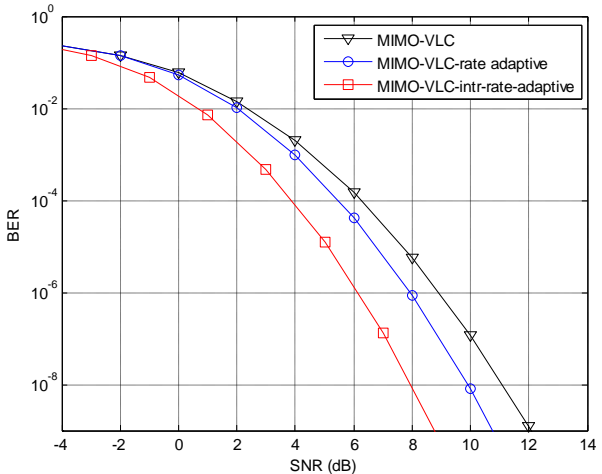

Figure 4 Simulation result of the proposed approach for $L=6$

Case III: $K=30 \mathrm{~dB}$, with Doppler shift $=500 \mathrm{~Hz}$ and AWGN noise of $10 \mathrm{~dB}$.

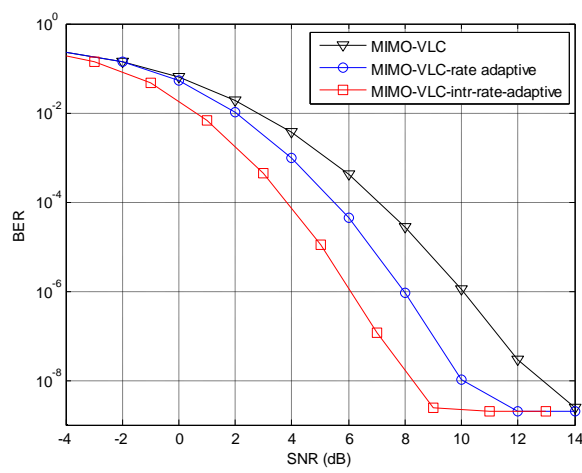

Figure 5 Simulation result of the proposed approach for $\mathrm{L}=3$

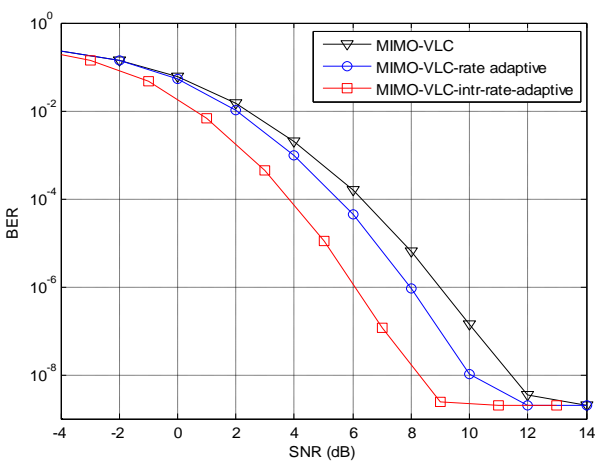

Figure 6 Simulation result of the proposed approach for $\mathrm{L}=6$ 
Case IV: $\mathrm{K}=30 \mathrm{~dB}$, with Doppler shift $=100 \mathrm{~Hz}$ and AWGN noise of $30 \mathrm{~dB}$.

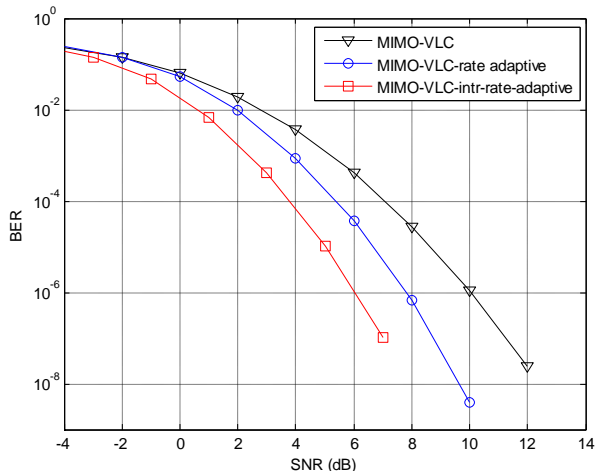

Figure 7 Simulation result of the proposed approach for $\mathrm{L}=3$

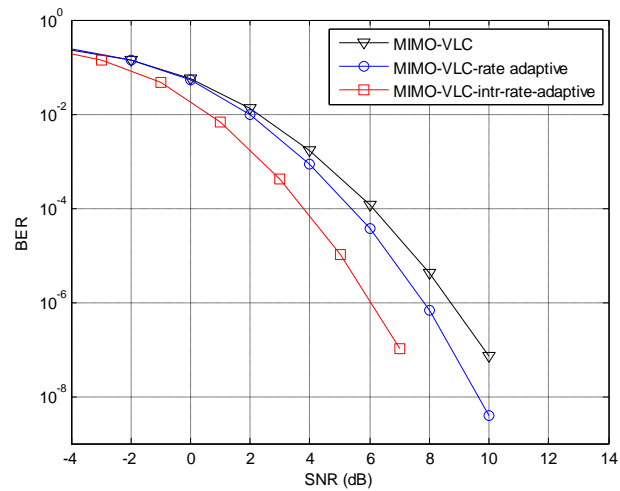

Figure 8Simulation result of the proposed approach for $\mathrm{L}=6$

Observations for different channel conditions with variation in block length $\mathrm{L}$ is shown in Figures 3 to 8 .

The observed BER is comparatively lower for higher values of $\mathrm{L}$ with proposed MIMO-VLC with weight 2-way LMS using bit sparse block coding.

\section{Conclusion}

A new mode of bit coding in interleave logic for block coding approach in MIMO-VLC system is proposed. The conventional coding of block coding, which allocate the transmitting bit based on the processing rate does not consider the bit coding as per the interference scenario. This limitation leads to higher error in receiver bits. The randomized channel scenario is applied by the governance of variance in SINR, where carrier allocation is controlled and bit allocation is monitored based on the variance in the channel SINR. The observed performance obtained for the proposed approach, illustrates an improvement of 0.0245 in BER performance for a given block length of 3 . Wherein the observed BER for the conventional mode is obtained to be 0.0234 . Under higher fading condition the BER is observed to be improved by 0.0112, and in Doppler shift variation this is observed to be 0.0236 . For higher noise level the BER obtained is observed to be reduced by 0.0023 compared to conventional approaches.

\section{References}

[1] MingFei Siyau, Tiancheng Li, Javier Prieto, Juan Corchado, Javier Bajo, “A Novel Pilot Expansion Approach for MIMO Channel Estimation and Tracking", IEEE,ICUWB, pp.1-5, 2015.

[2] Hani Mehrpouyan, Ali A. Nasir, Steven D. Blostein, Thomas Eriksson, George K. Karagiannidis, and Tommy Svensson, "Joint Estimation of Channel and Oscillator Phase Noise in MIMO Systems", IEEE Transactions on Signal Processing, Vol.60,(9),pp.4790 $-4807,2014$.

[3] Movahedian.A, McGuire, M., "Efficient and Accurate Semi blind Estimation of MIMO-OFDM Doubly-Selective Channels", 80" IEEE,VTC Fall, pp.1-5, 2014.

[4] S. D. Dissanayake and J. Armstrong, "Novel techniques for combating DC offset in diversity combined ACO-OFDM," IEEE Commun. Lett., vol. 15, no. 11, pp. 1237-1239, Nov. 2011.

[5] Q. Wang., "Layered ACO-OFDM for intensity-modulated direct-detection optical wireless transmission," Opt. Exp., vol. 23, no. 9, pp. 12382-12393, May 2015.

[6] M. S. Islim, D. Tsonev, and H. Haas, "Spectrally enhanced PAM-DMT for IM/DD optical wireless communications systems," in Proc. IEEE PIMRC, Hong Kong, Aug. 2015, pp. 877-882.

[7] Q. Wang, Z. Wang, and L. Dai, "Asymmetrical hybrid optical OFDM for visible light communications with dimming control," IEEE Photon. Technol. Lett., vol. 27, no. 9, pp. 974-977, May 2015. 
[8] D. J. F. Barros, S. K. Wilson, and J. M. Kahn, "Comparison of orthogonal frequency-division multiplexing and pulse amplitude modulation in indoor optical wireless links," IEEE Trans. Commun., vol. 60, no. 1, pp. 153-163, Jan. 2012.

[9] W. O. Popoola, Z. Ghassemlooy, and B. G. Stewart, "Pilot-assisted PAPR reduction technique for optical OFDM communication systems,” J. Lightw. Technol., vol. 32, no. 7, pp. 1374-1382, Apr. 2014.

[10] H. Qian., "On the benefit of DMT modulation in nonlinear VLC systems," Opt. Exp., vol. 23, no. 3, pp. 2618- 2632, Feb. 2015

[11] Rainfield Y Yen, Hong-Yu Liu and Chia-Sheng Tsai, "Iterative joint frequency offset and channel estimation for OFDM systems using first and second order approximation algorithms", EURASIP Journal on Wireless Communications and Networking, Vol.341, Springer, 2012.

[12] Marcello Cicerone, Osvaldo Simeone, and Umberto Spagnolini, "Channel Estimation for MIMO-OFDM Systems by Modal Analysis/Filtering”, IEEE transactions on communications, vol. 54, no. 11, IEEE, 2006.

[13] Te-Lung Kung and Keshab K Parhi, "Semi blind frequency-domain timing Synchronization and channel estimation for OFDM systems", EURASIP Journal on Advances in Signal Processing, Springer, 2013.

[14] Erol " Onen,1 AydinAkan (EURASIPMember), 1 and Luis F. Chaparro, "Time-Frequency Based Channel Estimation for HighMobility OFDM Systems-Part I:MIMO Case", Hindawi Publishing Corporation, EURASIP Journal on Advances in Signal Processing, pp.8, Springer, 2010

[15] Kyeong Jin Kim, Jiang Yue, Ronald A. Iltis, and Jerry D. Gibson, "A QRD-M/Kalman Filter-Based Detection and Channel Estimation Algorithm for MIMO-OFDM Systems", IEEE transactions on wireless communications, vol. 4, no. 2, IEEE, 2005.

[16] C Anjanaa, S Sundaresana, Tessy Zachariaa, R Gandhirajb, K P Soman, "An Experimental Study on Channel Estimation and Synchronization to Reduce Error Rate in OFDM Using GNU Radio", International Conference on Information and Communication Technologies, Procedia Computer Science, Vol.46, pp.1056 - 1063, Elsevier, 2015.

\section{AUTHOR'S PROFILE}

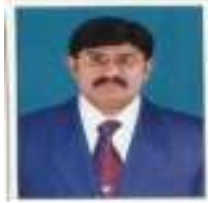

Dasari Subba Rao is a research Scholar from Rayalaseema University and working as Associate Professor Professor of ECE in Siddartha Institute of Engineering and Technology. He has done his Graduation in Engineering (ECE) in 2003 from JNTU, Hyderabad and Post Graduation in 2007 with specialization in Embedded Systems from SRM University, Chennai. He published 26 papers in International Journal, He is the life time member of ISTE. His area of interest is Wireless Communications.

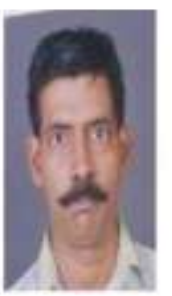

Dr.N.S.Murti Sarma belongs to K. Pedapudi , East Godavary district of Andhra Pradesh state, India2. He received his Model Diploma for Technicians(MDT), offered with collaboration of United Sates of Soviet Russia (USSR), with specialization in production of radio apparatus (RA) from Government Polytechnic of Masabtank, Hyderabad, his B.Tech from Jawharlal Nehru Technological University (JNTU) College of Engineering, Hyderabad in 1990, his M.E with specialization in Microwaves and Radar Engineering(MRE) from Osmania University in 1996 and his Ph.D in E.C.E from O.U, Hyderabad in 2002. As a Part of his diploma curriculum, He was at nuclear instruments division of instruments group in Electronics corporation of India limited (ECIL), Hyderabad as technician apprentice in 1984.

From 1991 to 1996 he was a lecturer to U.G courses in electronics, physics in faculty of science and various subjects of electronics and communications engineering for diploma holders (FDH) program of JNTU Engineering College at Hyderabad, and from 1996 to 2001 he was with R\&T Unit for navigational electronics (NERTU). During his association with NERTU, he executed projects sponsored by RCA, VSSC, DLRL and DST. His research interests include electromagnetic modeling, atmospheric studies, optical fiber communication, low power VLSI, signal processing. Several international and national publications are under his credit.

He continued his teaching from 2001 and currently at Sreenidhi Institute of Science and Technology as a professor of ECE. As one of the earlier assignments now he was Principal of SV Insititute of Technology and Engineering (SVIET) and professor of electroncs and communications Engineering of SV group of institutions. He teaching interests for undergraduate courses includes Electromagnetic theory, antennas and propagation and microwave engineering, post graduate courses in communication systems and microwave radar engineering. Dr. N.S.Murthy Sarma is life member of Institute of Science and Technology education (ISTE) since 2002 and fellow of institute of electronics and telecommunication engineers (IETE) since 2003, fellow of Institution of Engineers IE(I) and Member of Institute of Electrical and Electronics Engineers (IEEE) since 2010. He usually reviewers papers for international journals viz. international journal of computer science and Engineering systems and international journal of International Journal of Emerging Technologies and Applications in Engineering Technology and Sciences, besides a regular conference reviewer of conferences(since 2010) of IEEE with immediate recent assignment of ADVCIT'2014. . He is one of the recognized Ph.D Supervisors of engineering faculty, Around Eight research scholars are working with him under Ph.D. programme of JNTUH/JNTUK/KLU in the area of Communications, Low power VLSI, GPS/GLONASS, since 2008. 